\title{
La flore diatomique de quelques sédiments tourbeux holocènes des îles Kerguelen
}

\author{
B. Van de Vijver ${ }^{1,4}$ \\ L. Beyens ${ }^{1}$ \\ J.C. Gloaguen ${ }^{2}$ \\ Y. Frenot ${ }^{3}$
}

Mots clés: diatomées, tourbes, Kerguelen, Subantarctique

Cette étude de la flore diatomique subfossile dulçaquicole de la Grande Terre (Iles Kerguelen) a été réalisée à partir de six prélèvements effectués sur des dépôts tourbeux mis à jour après le retrait du Glacier Ampère, à l'ouest de l'archipel. Au total, 107 taxons de diatomées (84 espèces, 22 variétés et 1 forme) ont été observés. On constate une grande similitude entre cette flore subfossile, datant d'environ 1000, 5000 ou 10000 ans BP, et la flore diatomique actuelle des Iles Kerguelen. La biogéographie de cette flore et les similitudes avec la flore d'autres åles antarctiques sont brièvement discutées.

\section{Freshwater diatom flora from peaty sediments in Kerguelen Islands}

Keywords : diatoms, peat, Kerguelen, Subantarctic.

The freshwater diatom flora of The Grand Terre (Kerguelen Islands) is studied in six samples from Holocene peaty sediments, sampled after the retreat of the Ampère Glacier snout, in the western side of the archipelago. 107 taxa were observed (84 species, 22 varieties and 1 form). This diatom flora, about 1000,5000 and $10000 \mathrm{yr}$ old, showed a great similarity to the recent flora of the Kerguelen Islands. The biogeography of this flora and the similarities with other Antarctic islands is briefly discussed.

\section{Introduction}

Les Iles Kerguelen sont situées dans le sud de l'Océan Indien (Fig. 1), à plus de $4000 \mathrm{~km}$ des côtes africaines et australiennes. Les îles appartiennent à la

1. Université d'Anvers, Département de Biologie, Section Ecologie arctique et Paléobiologie, Groenenborgerlaan 171, B-2020 Anvers, Belgique.

2. UMR 6553 CNRS, Université de Rennes I, Laboratoire d'Ecologie Végétale, Campus de Beaulieu, F-35042 Rennes Cedex, France. 3. UMR 6553 CNRS, Université de Rennes I, Station Biologique de Paimpont, F-35380 Plélan-Le-Grand, France.

Auteur correspondant :

4. Bart Van de Vijver, University of Antwerp (RUCA), Department of Biology, Section Arctic Ecology, Limnology And Paleobiology, Groenenborgerlaan 171, B-2020 Antwerp, Belgium. province biogéographique dite subantarctique (Stonehouse 1982).

La flore algale de ces îles est bien connue. Thérézien (1976) et Thérézien \& Couté (1977) ont analysé la composition algale des eaux douces des Iles Kerguelen et Crozet, à l'exclusion des diatomées. Depuis l'étude des diatomées subfossiles de Germain (1937) sur quelques échantillons provenant des Iles Kerguelen, il y a eu peu d'études réalisées sur ce groupe, et pratiquement toutes, d'ordre taxonomique, ne concernent que la flore diatomique dulçaquicole récente. Les publications les plus importantes sont l'étude de Bourrelly \& Manguin (1954) et celles de Le Cohu \& Maillard (1983, 1986). D'autres études traitent d'observations plus ponctuelles sur quelques genres (Germain \& Le Cohu 1981, Le Cohu 1982, 1983). 


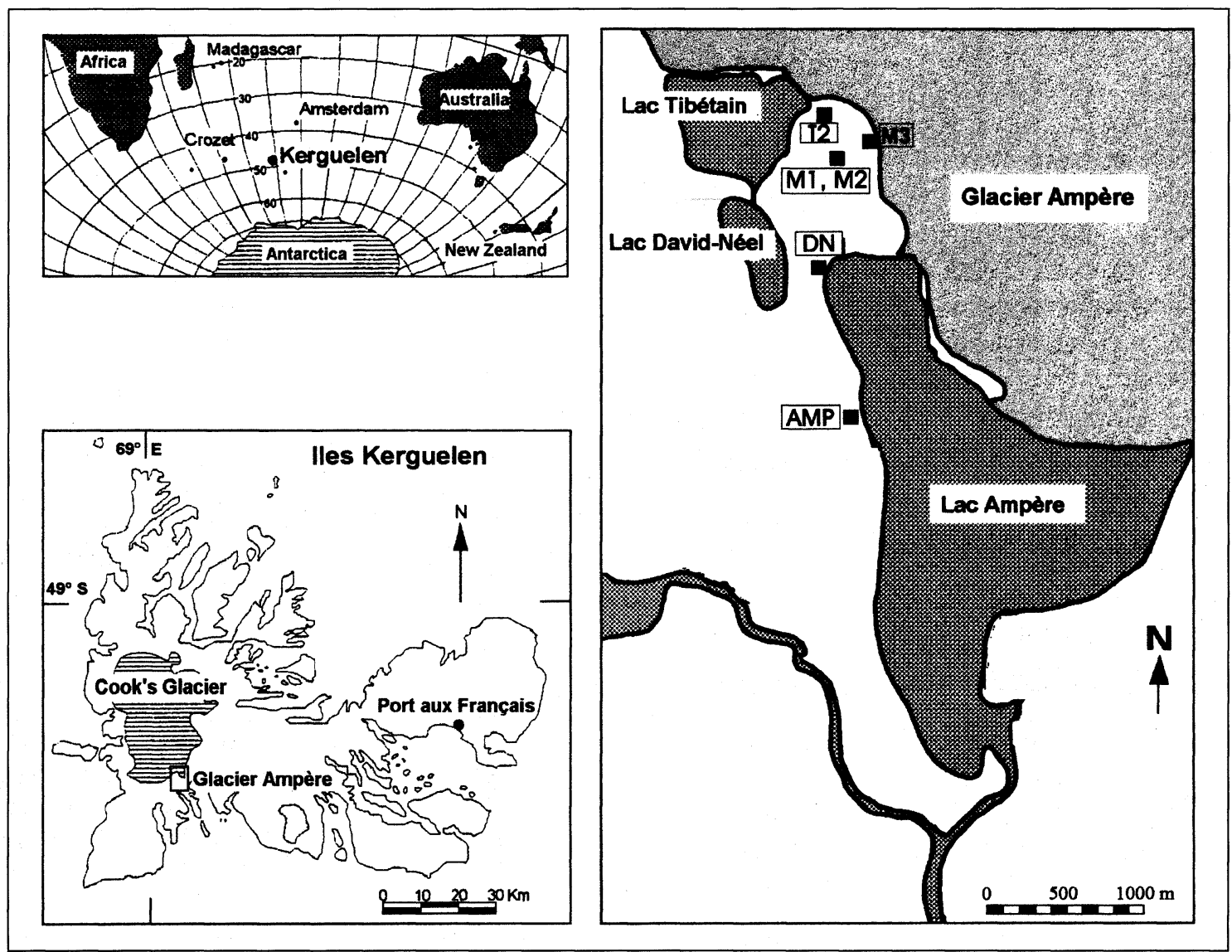

Fig. 1. Carte schématique de la localisation des sites d'échantillonnages (d'après Frenot et al. 1997).

Fig. 1. Sketch map of the study site with the locations of the different sampling points (after Frenot et al. 199\%),

La découverte de sédiments tourbeux après le retrait du Glacier Ampère, dans l'ouest de Kerguelen, nous a permis d'étudier la flore diatomique subfossile de ce secteur de l'archipel. Cette flore a été comparée à celle déjà mentionnée dans la littérature, soit à Kerguelen, soit dans d'autres îles subantarctiques.

\section{Matériel et Méthodes}

Depuis le milieu des années 1970, le front du Glacier Ampère, l'un des principaux émissaires de la Calotte Cook, a reculé de façon très rapide sur une distance de plus de $3 \mathrm{~km}$ (Frenot et al. 1993) (Fig. 1). En février 1995, six échantillons ont été prélevés des sédiments tourbeux pour faire l'objet d'une datation au $14 \mathrm{C}(\mathrm{Be}-$ ta Analytic Inc., USA). Le Tableau 1 résume les résultats de ces datations (Frenot et al. 1997). Une étude des diatomées présentes dans ces prélèvements a également été réalisée. Pour ce faire, les échantillons ont été oxydés par $\mathrm{H}_{2} \mathrm{O}_{2}$ et $\mathrm{KMnO}_{4}$ (Van der Werff 1955). Les diatomées traitées ont ensuite été montées dans du Naphrax. Un minimum de 500 valves a été dénombré et identifié sur chaque lame en utilisant un microscope Leitz Orthoplan équipé avec une optique à contraste de phase. On a ainsi pu estimer les fréquences relatives des principaux taxons. Les taxons plus rares ont été observés en examinant toutes les lames avec un fort grossissement. La nomenclature adoptée repose sur les travaux de Krammer \& Lange-Bertalot (1986-1991) et de Lange-Bertalot (1993). Pour le genre Pinnularia, Krammer (1992) a été employé. Comme indice de similarité, l'Indice de Communauté de Sørensen (1948) a été utilisé : $2 c /(a+b+2 c)$ 
Tableau 1. Echantillons tourbeux analysés dans cette étude. Epoques de mise à jour de ces dépôts et datations absolues au 14C (d'après Frenot et al 1997).

Table 1. Peat samples used in this study. Dates of appearance of these organic deposits and 14C age (after Frenot et al. 1997).

\begin{tabular}{|c|c|c|c|c|c|c|}
\hline Echantillon & [Code] & $\begin{array}{c}\text { Référence } \\
\text { Beta }\end{array}$ & $\begin{array}{l}\text { Altitude } \\
\text { (m) }\end{array}$ & $\begin{array}{l}\text { Macrorestes } \\
\text { (dominants) }\end{array}$ & Mise à jour & Age \\
\hline Ampère & [AMP] & Beta- 82425 & 160 & Azorella selago & $1975-1980$ & $900 \pm 70 \mathrm{BP}$ \\
\hline David-Néé 4 & {$[\mathrm{DN}]$} & Beta-82419 & 30 & Acaena magellanica & $1980-1985$ & $1320 \pm 70 \mathrm{BP}$ \\
\hline Tibétain 2 & [T2] & Beta-82424 & 240 & Mousses & 1992 & $4590 \pm 60 \mathrm{BP}$ \\
\hline Mongole 1 & [M1] & Beta- 82420 & 260 & $?$ & 1990 & $10140 \pm 120 \mathrm{BP}$ \\
\hline Mongole 2 & [M2] & Beta-82421 & 265 & Mousses & 1990 & $10220 \pm 90 B P$ \\
\hline Mongole 3 & [M3] & Beta-82422 & 280 & Mousses & 1994 & $9930 \pm 70 B P$ \\
\hline
\end{tabular}

$a \& b=$ nombre d'espèces présentes uniquement dans les deux sites

$\mathrm{c}=$ nombre d'espèces communes aux deux sites).

\section{Résultats et discussion}

Une flore diatomique très riche est observée dans quatre des six échantillons analysés. Seuls les échantillons de T2 et M3 montrent une flore moins diversifiée. La flore de $\mathrm{M} 3$ est même très pauvre puisque près de $75 \%$ des valves observées appartiennent à cinq espèces uniquement.

Au total 107 taxons ont été dénombrés (Tableau 2), dont 84 espèces, 22 variétés et 1 forme, qui se répartissent parmi 22 genres (Tableau 3). Les genres les plus couramment observés sont Achnanthes (17\% de tous les taxons observés), Pinnularia (17\%) et Navicula (14 \%). Deux espèces (Fragilariopsis angulata Hasle et $F$. kerguelensis (O'Meara) Hasle) sont d'origine marine et ne sont probablement que des contaminants du milieu marin. Mis à part quelques taxons tels que Achnanthes abundans Manguin, $A$. confusa Manguin, $A$. alteragracillima (Meister) Lange-Bertalot, A. aueri Krasske, Fragilaria exigua Grunow et Diatomella hustedti Manguin qui ont une abondance relative supérieure à $5 \%$, la plus grande partie des taxons trouvés sont très faiblement représentés. Ceci rejoint les observations de Oppenheim (1990) et de Le Cohu \& Maillard (1983), qui ont constaté que la majorité des diatomées doit vivre aux Kerguelen dans des biotopes moins favorables.

L'analyse quantitative des diatomées (Fig. 2) montre qu'Achnanthes est le genre le plus couramment observé avec $51 \%$ de toutes les valves comptées, suivi par Navicula $(11,2 \%)$ et Pinnularia $(9,5 \%)$. Cette dominance totale de Achnanthes a souvent été observée dans les eaux douces antarctiques (Oppenheim 1994, Van de Vijver \& Beyens 1996) et peut être expliquée,' d'une part par leur préférence pour des eaux oligotrophes et proches de la neutralité, très communes en milieu subantarctique (Pierre 1967), d'autre part par la présence de pierres et de végétation souvent humides (mousses terrestres presque immergées, végétation herbeuse maintenue constamment humide par les précipitations abondantes et régulières...), qui constituent un habitat idéal pour les espèces rattachées au genre Achnanthes (Lange-Bertalot \& Krammer 1989, Bjîrck et al. 1991). En Géorgie du Sud par exemple, nous avons pu observer une communauté diatomique à Achnanthes incognita - Fragilaria germainii sur des mousses gorgées d'eau mais non immergées (Van de Vijver \& Beyens 1997. La composition diatomique de

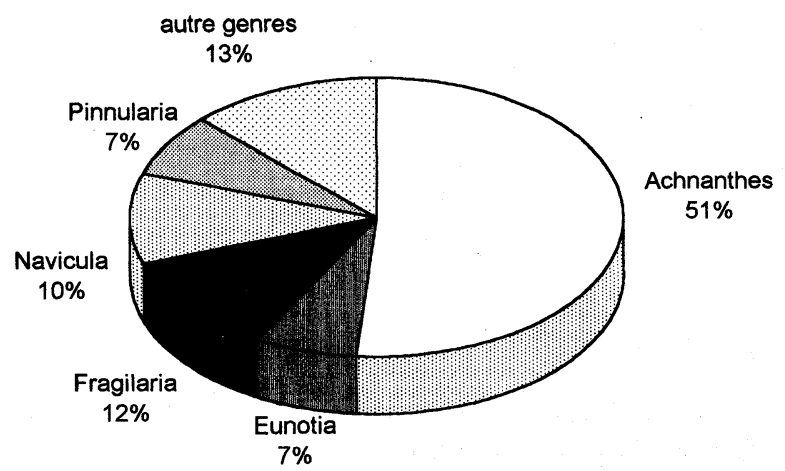

Fig. 2. Importance relative des principaux genres de diatomées observés dans les sédiments organiques découverts récemment par le retrait du glacier Ampère.

Fig. 2. Relative importance of the main diatom genera, found in this study. 
Tableau 2. Liste des taxons observés dans cette étude. $\mathrm{A}$ = espèce indigène (sub-)antarctique, $\mathrm{C}=$ cosmopolite, $\mathrm{K}=$ espèce endémique aux Kerguelen, $S$ = espèce de l'hémisphère austral.

Table 2 . List of all diatoms observed in this study. $\mathrm{A}=$ sub-antarctic species, $\mathrm{C}=$ cosmopolitan, $\mathrm{K}=$ endemic species on the Kerguelen , $\mathrm{S}=$ species of the southern hemisphere.

Liste des taxons

Achnanthes abundans Manguin

Achnanthes alteragracillima (Meister) Lange-Bertalot

Achnanthes aueri Krasske

Achnanthes confusa Manguin

Achnanthes helvetica (Hust.) Lange-Bertalot

Achnanthes incognita Krasske

Achnanthes aff. laevis var. diluviana (Hust.) Lange-Bertalot

Achnanthes laevis var. ninkei (Guer. \& Man.) Lange-Bertalot

Achnanthes lanceolata (Bréb.) Grunow

Achnanthes lanceolata var. lanceolatoides (Sov.) Reimer

Achnanthes manguini i Hustedt

Achnanthes manguinii var. elliptica Manguin

Achnanthes minutissima Kutzing var. minutissima

Achnanthes modestiformis Lange-Bertalot

Achnanthes oblongella Oestrup

Achnanthes sp1

Achnanthes stauroneioides Manguin

Achnanthes subatomoides (Hust.) Lange-Bertalot

Amphora libyca Ehrenberg

Aulacoseira sp1

Brachysira exilis (Kotz.) Round \& Mann

Caloneis aff. tenuis (Gregory) Krammer

Caloneis bacillum (Grun.) Cleve

Caloneis marnieri Manguin

Caloneis silicula (Ehr.) Cleve

Caloneis sp1

Cymbella aff. lapponica Grunow

Cymbella incerta (Grun.) Cleve

Cymbella kerguelensis Germain

Cymbella silesiaca Bleisch

Cymbella sp1

Diatomella hustedtii Manguin

Diploneis subovalis Cleve

Eunotia aff. exigua (Bréb.) Grunow

Eunotia arcubus Nörpel \& Lange-Bertalot

Eunotia monodon var. major (W. Sm.) Hustedt

Eunotia muscicola var. tridentula Nörpel \& Lange-Bertalot

Eunotia nymanniana Grunow

Eunotia paludosa Grunow var. paludosa

Eunotia praerupta Ehrenberg

Eunotia praerupta var. curta Grunow

Eunotia sp1

Fragilaria bicapitata Mayer

Fragilaria construens $f$. venter (Ehr.) Hustedt

Fragilaria exigua Grunow

Fragilaria germainii Reichardt \& Lange-Bertalot

Fragilaria leptostauron (Ehr.) Hustedt var. leptostauron

Fragilaria maillardi Le Cohu

Fragilaria pinnata Ehrenberg var. pinnata

Fragilaria vaucheriae var. tenuis Manguin

Fragilariopsis angulata Hasle

Fragilariopsis kerguelensis (O'Meara) Hasle

Frustulia pulchra Germain

Frustulia rhomboides (Ehr.) De Toni var. rhomboides
Status

A

C

A

A

C

S

C

A

C

A

A

A

C

C

C

A

A

C

C

C

C

A, $\mathrm{K}$

C

C

C

A, $\mathrm{K}$

C

A

C

C

C

C

C

C

C

C

c

C

C

A

A

C

$A, K$
$C$

C

A

A

A, $K$

$\mathrm{C}$
Liste des taxons

Gomphonema affine Kutzing

Gomphonema sarcophagus Gregory

Gomphonema signyensis Kociolek \& Jones

Gomphonema sp1

Hantzschia amphioxys (Ehr.) Smith

Navicula arcuata Heiden

Navicula bicephala Hustedt

Navicula bryophila Petersen

Navicula bryophiloides Manguin

Navicula fossalis Krasske var. fossalis

Navicula geniculata Germain

Navicula ignota var. palustris Hustedt

Navicula jaagii Meister

Navicula linearis (O. Maller) Frenguelli

Navicula portomonttana Cleve

Navicula pseudoscutiformis Hustedt

Navicula soehrensis var. muscicola (Pet.) Krasske

Navicula sp1

Navicula sp2

Navicula sp3

Neidium aubertii Manguin

Neidium bisulcatum (Lagerstedt) Cleve

Nitzschia ignorata Krasske

Nitzschia perminuta (Grun.) Peragalio

Nitzschia sp1

Orthoseira roeseana (Rabenhorst) O'Meara

Pinnularia 8

Pinnularia acoricola Hustedt

Pinnularia aff. anglica Krammer

Pinnularia anglica Krammer

Pinnularia borealis var. cuneorostrata Manguin

Pinnularia borealis var. scalaris (Ehr.) Rabenhorst

Pinnularia borealis var. thuringiaca (Rab.) Krammer

Pinnularia circumducta Manguin

Pinnularia divergentissima (Grun.) Cleve

Pinnularia microstauron (Ehr.) Cleve

Pinnularia microstauron var. elongata Manguin

Pinnularia obscura Krasske

Pinnularia rupestris Hantzsch

Pinnularia silvatica Petersen

Pinnularia similiformis Krammer

Pinnularia sp1

Pinnularia sp2

Pinnularia viridiformis Krammer

Rhopalodia rupestris (W. Sm.) Krammer

Stauroneis alpina Hustedt

Stauroneis anceps var. sibirica Grunow

Stauroneis javanica (Grun.) Cleve

Stauroneis kriegeri Patrick

Stauroneis phoenicenteron (Nitzsch) Ehrenberg

Surirella angusta Kutzing

Synedra rumpens var. familiaris (Kutz.) Grunow
C

C

C

A

C

C

A, K

C

A, K

C

A

C

C

A

C

A

C

C

A

A, K

C

C

A

C

C

C

C

C

C

C

Nombre de taxons (sub-)antarctiques (total $=27$ )

Nombre de taxons (total $=107$ ) 
Tableau 3. Nombre de taxons dans chacun des genres présents dans les sédiments organiques étudiés.

Table 3. Number of recorded taxa in each genus.

\begin{tabular}{lc}
\hline Achnanthes & 18 \\
Pinnularia & 18 \\
Navicula & 15 \\
Eunotia & 9 \\
Fragilaria & 8 \\
Caloneis & 5 \\
Cymbella & 5 \\
Nitzschia & 5 \\
Stauroneis & 5 \\
Gomphonema & 4 \\
Frustulia & 3 \\
Neidium & 2 \\
Amphora & 1 \\
Aulacoseira & 1 \\
Brachysira & 1 \\
Diatomella & 1 \\
Diploneis & 1 \\
Hantzschia & 1 \\
Orthoseira & 1 \\
Rhopalodia & 1 \\
Surirella & 1 \\
Synedra & 1 \\
Total & 107 \\
\hline
\end{tabular}

certains des échantillons de Kerguelen (A et M2) montrent d'ailleurs une assez grande similitude avec cette communauté associée aux bryophytes de Géorgie du Sud, surtout en ce qui concerne les espèces du genre Achnanthes.

Le nombre des espèces endémiques antarctiques est relativement réduit. $22 \%$ des taxons observés ont déjà été cités dans la littérature comme étant des espèces indigènes pour la région-(sub-)antarctique (Tableau 2). Ces taxons autochtones ne représentent d'ailleurs que $10 \%$ des valves dénombrées. Ce sont donc les éléments cosmopolites qui constituent la plupart des diatomées d'eau douce des abords du Glacier Ampère. Cette observation est en accord avec les autres études sur les diatomées d'eau douce des îles (sub-)antarctiques. Par exemple, Van de Vijver \& Beyens (1996) ont montré que le nombre des diatomées (sub-)antarctiques présentes dans les communautés dulçaquicoles de Géorgie du Sud atteint à peine $9 \%$. Hirano (1965) constatait également que près de $50 \%$ des diatomées trouvées dans la région antarctique ont une plus vaste distribution mondiale que le simple domaine polaire ou subpolaire austral.

\section{Biogéographie}

Les résultats présentés dans cette étude sont comparés avec la composition diatomique d'autres îles des régions subantarctique et antarctique telles qu'elles sont définies par Stonehouse (1982) d'après leurs caractéristiques climatiques et écologiques.

La flore diatomique des Iles Kerguelen est maintenant évaluée à 345 taxons. Les principaux taxons nouveaux sont illustrés sur la Fig. 3. Les résultats obtenus sur la flore subfossile des abords du glacier Ampère montrent beaucoup de similitudes avec ceux de Bourrelly \& Manguin (1954) et ceux de Le Cohu \& Maillard (1983, 1986) sur la flore diatomique récente des Iles Kerguelen. Parmi les 107 taxons que nous avons pu observer, 49 sont également cités par Le Cohu \& Maillard $(1983,1986)$ et 42 par Bourrelly \& Manguin (1954). Vingt-sept taxons sont considérés antarctiques (Tableau 4), et 7 sont jugés endémiques des Iles Kerguelen par Le Cohu $(1982,1983)$. Cependant, Pinnularia microstauron var. elongata Manguin a également été observé par Oppenheim (1990) à l'Ile Signy et par les auteurs dans la région de la Péninsule antarctique (Van de Vijver \& Beyens 1997). Aussi, dans l'état actuel de nos connaissances, six des taxons récoltés dans les tourbes des abords du Glacier Ampère peuvent être considérés comme endémiques vrais de Kerguelen.

Cette flore diatomique subfossile est également assez proche de celle décrite par Germain (1937). Celuici dénombre 34 espèces dont 12 sont également présentes dans la flore de nos échantillons tourbeux (Tableau 4). Cet auteur souligne que le genre Pinnularia est bien représenté en nombre de taxons, mais assez mal en nombre d'individus . Cela est aussi le cas dans la présente étude puisque, globalement, $17 \%$ des taxons appartiennent à ce genre mais ceux-ci ne sont représentés que par $9,5 \%$ des individus. Il y a néanmoins deux différences notables entre les deux études : Germain (1937) n'a trouvé aucun taxon du genre $\mathbf{E u}$ notia, présents au nombre de 9 ici, et la dominance du genre Achnanthes n'est pas aussi prononcée dans ses résultats que dans les nôtres.

La zone subantarctique tempérée froide à laquelle appartient l'archipel de Kerguelen (Stonehouse 1982) contient également des Iles telles que Crozet, Marion, Macquarie et Campbell. La confrontation de nos résultats avec ceux de la littérature sur ces îles permet de comparer la composition de leurs flores diatomiques (Tableau 4). Ainsi, Pierre (1977) a dénombré sur les îles Crozet 77 espèces, variétés et formes dont environ $16 \%$ figurent également dans la liste de flore subfossile analysée ici. Une seule étude a été faite sur Camp- 
Tableau 4. Comparaison entre cette étude et d'autres de la région subantarctique. L'indice de Communauté (Sørensen 1948) est utilisé comme indice de similarité.

Table 4. Comparison between this study and other studies in the subantarctic region. The Community Index Sørensen 1948) is used as a measurement of similarity.

\begin{tabular}{lcccc}
\hline Sites & Références & \# genres & \# espèces & $\begin{array}{c}\text { similarité } \\
\text { avéc cette étude }\end{array}$ \\
\hline Kerguelen & Germain (1937) & 16 & 33 & 0,17 \\
Kerguelen & Bourrelly \& Manguin (1954) & 27 & 160 & 0,31 \\
Kerguelen & Le Cohu \& Maillard (1983, 1986) & 35 & 279 & 0,25 \\
Kerguelen & cette étude & 22 & 107 & - \\
Campbell Island & Hickman \& Vitt (1973) & 23 & 59 & 0,06 \\
Crouzet & Pierre (1977) & 23 & 77 & 0,16 \\
Macquarie & Bunt (1954) & 24 & 60 & 0,12 \\
Marion Island & O'Meara (1876) & 4 & 5 & voir texte \\
\hline
\end{tabular}

bell Island (Hickman \& Vitt 1973). Ces auteurs y font état de 59 espèces, appartenant à 23 genres et l'indice de similarité de Sørensen (1948), comparant cette communauté et la nôtre, est très faible (Tableau 4). Il faut cependant remarquer que l'étude de Hickman \& Vitt (1973) concernait uniquement les diatomées terrestres et épiphytiques. Pour l'île Marion, nous ne disposons que des données de O'Meara (1876) ; cette étude, très ancienne, ne mentionne que cinq espèces de diatomées dont aucune n'est présente dans notre échantillonnage. Bunt (1954) a dénombré 60 espèces sur l'île Macquarie dont 10 espèces sont également présentes dans notre étude. Son étude est basée non seulement sur des échantillons récents (provenants des lacs et des sols) mais aussi des tourbes et des dépôts fluvio-glaciaires.

Nous disposons de nombreuses données sur la flore diatomique de Géorgie du Sud, l'une des îles de la Province Périantarctique de Stonehouse (1982). Des similitudes entre cette flore (Van de Vijver \& Beyens 1996, 1997) et celle de notre étude apparaissent clairement mais surtout à travers leurs éléments cosmopolites. Il n'y a en effet que 10 taxons, sur les 44 communs, qui sont indiqués indigènes de la région subantarctique. La prise en compte des études de Carlson (1913) et Fukushima (1965) permet d'aboutir aux mêmes conclusions.

En revanche, il y a peu de taxons communs entre ceux que nous avons obervés à Kerguelen et la flore des îles de l'Antarctique maritime telles que les Iles Signy (e.g. Oppenheim \& Greenwood 1990, Jones 1993), Livingston (e.g. Priddle \& Dartnall 1978) et King George (Schmidt et al. 1990, Martinez-Macchiavello et al. 1996), qui sont plus proches de la Péninsule Antarctique (Hansson \& Håkansson 1992, Wasell \& Håkansson, 1992. Quelques espèces communes existent bien, mais comme en Géorgie du Sud, ce sont seulement des taxons de type cosmopolite. Le genre Achnanthes, auquel appartiennent un grand nombre de taxons subantarctiques, disparaît presque totalement en s'éloignant vers le sud. Une étude de la flore diatomique de la région de la Péninsule Antarctique montre que Achnanthes ne constitue que $3 \%$ des valves observées. A l'inverse, des genres tels que Luticola (notamment Luticola muticopsis Van Heurck), Pinnularia et Nitzschia sont dominants dans cette région (Van de Vijver \& Beyens 1997).

Fig. 3. Photos au microscope photonique de quelques taxons nouveaux pour les Kerguelen : 1 Aulacoseira alpigena, 2 Fragilariopsis angulata, 3 Fragilaria exigua, 4 Eunotia arcubus, 5 E. muscicola var. tridentula, 6 E. nymanniana, 7 E. praerupta var. curta, 8 Achnanthes laevis var. inflata, valve à raphé , 9 A. oblongella valve sans raphé, 10 Navicula fossalis var. fossalis, $11 \mathrm{~N}$. soehrensis var. muscicola, $12 \mathrm{~N}$. jaagii, 13 Cymbella incerta, 14 C. lapponica, 15 C. silesiaca, 16 Frustulia rhomboides var. amphipleuroides, 17 Pinnularia viridiformis, 18 P. borealis var. thuringiaca, 19 P. silvatica, 20 P. divergentissima, 21 Pinnularia sp., 22 Caloneis tenuis, 23 Gomphonema signyensis, 24 Stauroneis alpina, 25 Stauroneis sp., 26 Rhopalodia rupestris. Le trait d'échelle indique $10 \mu \mathrm{m}$.

Fig. 3 : Light microscopical pictures of some diatoms, new for the Kerguelen Islands : Scale bar indicates $10 \mu \mathrm{m}$. 

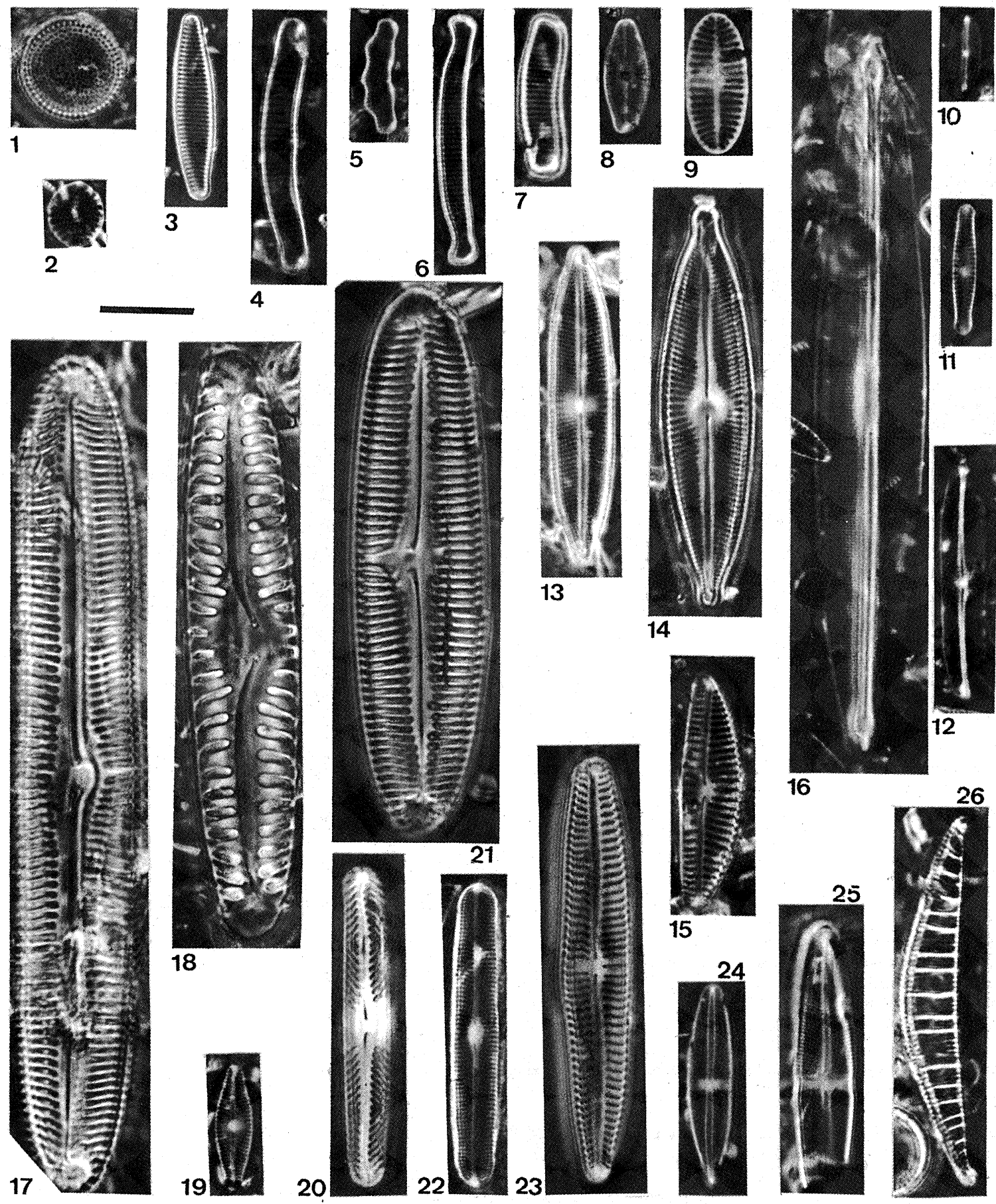

13
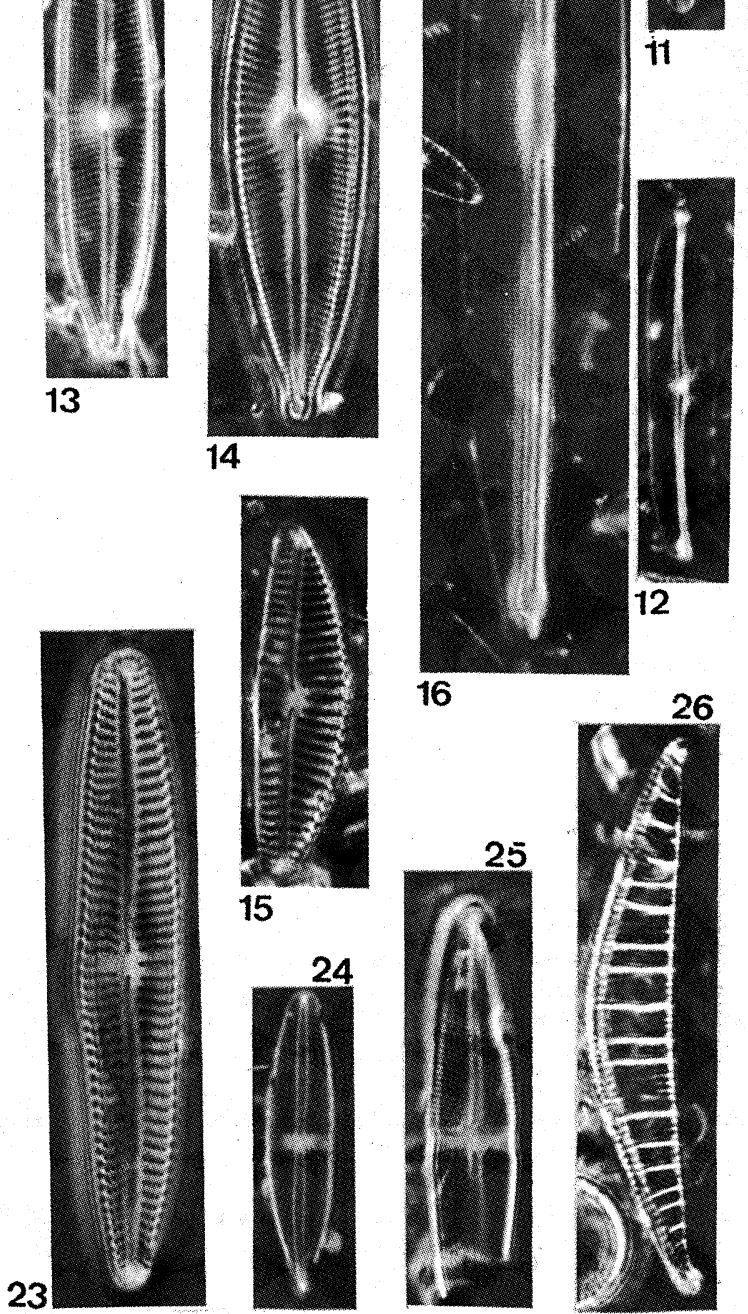

16

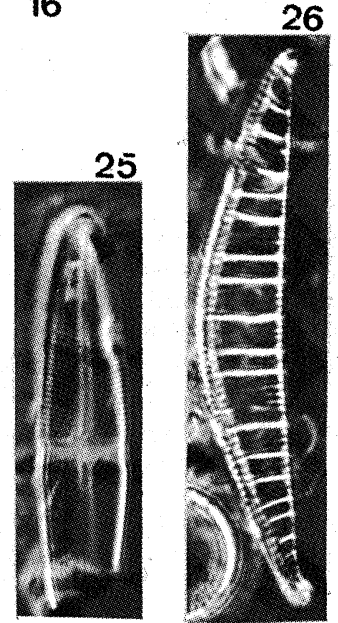




\section{Conclusion}

La flore diatomique holocène des sédiments organiques étudiés, qui compte plus d'une centaine de taxons, est particulièrement riche. Elle présente de nombreuses similitudes, d'une part avec celle, subfossile, décrite par Germain (1937), et d'autre part avec la flore actuelle (Bourrelly \& Manguin 1954, Le Cohu \& Maillard, 1983, 1986).

La comparaison des flores des îles de l'Océan Indien sud avec celles d'autres îles circumpolaires de l'Océan Atlantique sud suggère qu'il existe d'assez grandes similitudes entre les îles appartenant à la province subantarctique (Kerguelen, Crozet) mais qu'en revanche, cette flore se distingue assez nettement des îles situées dans la région antarctique maritime, près de la Péninsule Antarctique (Signy, Livingston, King George) et même de la flore sur la Géorgie du Sud, appartenant à la province péri-antarctique. Hirano (1965) considère que du point de vue de leur flore diatomique, les îles circum-antarctiques appartiennent à une seule et unique province biogéographique, dite Subantarctica. Il est clair, au vu de nos résultats, que ce regroupement est abusif. En revanche, la zonation écologique proposée par Stonehouse (1982), distinguant notamment les provinces subantarctique, périantarctiques et antarctique maritime, semble être en adéquation avec les caractéristiques biogéographiques des diatomées australes.

\section{Remerciements}

Les auteurs remercient le Dr. L. Denys pour la vérification de quelques diatomées. Bart Van de Vijver est aspirant-chercheur de la Fondation de Recherches Scientifiques (FWO), Flandres. Les prélèvements de tourbes ont été effectués dans le cadre du Programme d'Ecologie Terrestre $n^{\circ} 136$ de l'Institut Français pour la Recherche et la Technologie Polaires.

\section{Travaux cités}

Björck S., HÜkansson H., Zale R., Karlen W. \& Jönsson B.L. 1991. - A late Holocene lake sediment sequence from Livingston Island, South Shetland Islands, with palaeoclimatic implications. Antarct. Sci., $31:$ 61-72.

Bourrelly P. \& Manguin E. 1954. - Contribution à la flore algale d'eau douce des Iles Kerguelen. Mém. Inst. Sc. Madagascar, sér. B $5: 7-58$.

Bunt J.S. 1954. - A comparative account of the terrestrial diatoms of Macquarie Island. Proc. Lin. Soc. New South Wales, LXXIX 1$2: 34-57$.

Carlson G.W.F. 1913. - Süsswasseralgen aus der Antarktis, SüdGeorgien und den Falkland Inseln. Wiss. Erg. Schwed. Sîdpolar Exped., IV : 1-94.

Frenot Y., Gloaguen J.C., Picot G., Bougère J., Benjamin D. 1993. -Azorella selago Hook. used to estimate glacier fluctuations and climatic history in the Kerguelen Islands over the last two centuries. Oecologia, $95: 140-144$.
Frenot Y., Gloaguen J.-C., Van de Vijver B. \& Beyens L. 1997. Datation de quelques sédiments tourbeux holocènes et oscillations glaciaires aux îles Kerguelen. C. R. Acad. Sci. Paris, 320 : 567-573.

Fukushima H. 1965. - Preliminary report on diatoms from South Georgia. Antarct. Rec., 24 : 18-30.

Germain H. 1937. - Diatomées d'une tourbe des Iles Kerguelen. Bull. Soc. Fr. Microsc. VI1 : 11-17.

Germain H. \& Le Cohu R. 1981. - Variability of some features in a few species of Gomphonema from France and the Kerguelen Islands South Indian Ocean. Proc. 6th Symp. recent and fossil Diatoms, Budapest 1980, Koeltz, Koenigstein : 167-178.

Hansson L.-A. \& Håkansson H. 1992. - Diatom community response along a productivity gradient of shallow Antarctic lakes. Pol. Biol., 12 : 463-468.

Hickman M. \& Vitt D.H. 1973. - The aerial epiphytic diatom flora of moss species from subantarctic Campbell Island. Nov. Hedw., 24 2-4 : 443-458.

Hirano M. 1965. - Freshwater algae in the Antarctic regions. Monogr. Biol., 15, 127-193.

Jones V. 1993. - The use of diatoms in lake sediments to investigate environmental history in the Maritime Antarctic: an example from Sombre Lake, Signy Island. Ant. Spec. Topic : 91-95.

Krammer K. \& Lange-Bertalot H. 1986-1991. - Süsswasserflora von Mitteleuropa Bacillariophyceae Teil 1-4. G. Fisher-Verlag, Stuttgart : $2486 \mathrm{p}$.

Krammer K. 1992. - Pinnularia. Eine monographie der EuropÑischen Taxa. Bibliotheca Diatomologica J. Cramer Ed., BerlinStuttgart. Band $26: 1-353$.

Lange-Bertalot H. \& Krammer K. 1989. - Achnanthes, eine Monographie der Gattung. Bibliotheca Diatomologica,J. Cramer Ed. Berlin-Stuttgart. Band 18 : 1-393.

Lange-Bertalot H. 1993. - 85 neue Taxa und über 100 weitereneu definierte Taxa ergänzend zur Süsswasserflora von Mitteleuropa Vol 2/1-4. Bibliotheca Diatomologica J. Cramer Ed., Berlin-Stuttgart. Band $27: 453 \mathrm{p}$.

Le Cohu R. 1982. - Les espèces endémiques de Diatomées d'eau douce aux Iles Kerguelen. C.N.F.R.A. 51 : 35-42.

Le Cohu R. 1983. - Observations sur deux espèces de Diatomées du genre Diatomella : Diatomella hustedtii Manguin et Diatomella ouenkoana Maillard. Crypt. Algol. 41/2 : 63-71.

Le Cohu R. \& Maillard R. 1983. - Les diatomées monoraphidées des Iles Kerguelen. Annls. Limnol. 193 : 143-167.

Le Cohu R. \& Maillard R. 1986. — Diatomées d'eau douce des Iles Kerguelen à l'exclusion des Monoraphidées. Annls. Limnol. 222 : 99-118.

Martinez-Macciavello J.C., Tatur A., Servant-Vildary S. \& Del Valle R. 1996. - Holocene environmental change in a marine-estuarine-lacustrine sediment sequence, King George Island, South Shetland Islands. Antarct. Sci. 84 : 313-322.

O'Meara E. 1876. - On the diatomaceous gatherings made at Kerguelen'Land by H.N. Moseley, H.M.S. Challenger. J. Lin. Soc., Bot. XV : 55-59.

Oppenheim D.R. 1990. - A preliminary study of the benthic diatoms in contrasting lake environments. In : Antarctic ecosystems. Ecological change and conservation, K.R. Kerry \& G. Hempel. Springer-Verlag, Berlin (Ed.) : 91-99.

Oppenheim D.R. 1994. - Taxonomic studies of Achnanthes Bacillariophyta in freshwater maritime antarctic lakes. Can. J. Bot. 72 : 1735-1748.

Oppenheim D.R. \& Greenwood R. 1990. - Epiphytic diatoms in two freshwater maritime antarctic lakes. Freshwater. Biol. 24 : 303-314. 
Pierre J.-F. 1967. — Introduction à l'étude du phytoplancton des formation d'eau douce des Terres Australes Antarctiques Françaises. T.A.A.F., Paris $41: 22-25$.

Pierre J.-F. 1977. — Les algues des eaux courantes de l' le de la Possession Archipel Crozet. Bull. Soc. Phycol. Fr., 22 : 79-86.

Priddle J. \& Dartnall H.J.G. 1978. - The biology of an Antarctic moss community. Freshwater. Biol. $8: 469-480$.

Schmidt R., Mäusbacher R. \& Müller J. 1990. — Holocene diatom flora and stratigraphy from sediment cores of two Antarctic lakes King Georg Island. J. Paleolimn. 3 : 55-74.

Sørensen T. 1948. - A method of establishing groups of equal amplitude in plant sociology based on similarity of species content. Det. Kong. Danske. Vidensk. Selsk. Biol. Skr. (Copenhagen), 54 : $1-34$.

Stonehouse B. 1982. - La zonation écologique sous les hautes latitudes australes. C.N.F.R.A. $51: 532-537$.

Thérézien Y. 1976. - Algues d'eau douce des Iles Kerguelen et Crozet à l'exclusion des Diatomées. C.N.F.R.A. 41 : 21-59.
Thérézien Y. \& Couté A. 1977. - Algues d'eau douce des Iles Kerguelen et Crozet à l'exclusion des Diatomées. C.N.F.R.A. 43 : 191.

Van der Werff A. 1955. - A new method of concentrating and cleaning diatoms and other organisms. Verh. Int. Verein. Theor. Angew. Limnol. 12 : 276-277.

Van de Vijver B. \& Beyens L. 1996. - Freshwater diatom communities of the Stromness Bay area, South Georgia. Antarct. Sci. 84 : 359-368.

Van de Vijver B. \& Beyens L. 1997. - The epiphytic diatom flore from mosses of Stromness Bay area. Polar Biology. 17 : 492-501.

Van de Vijver B. \& Beyens L. soumis. - A preliminary study of freshwater diatoms of small islands in the Maritime Antarctic Region. Antarct. Sci.

Wasell A. \& Håkansson H. 1992. - Diatom stratigraphy in a lake on Horseshoe Island, Antarctica : a marine-brackish-freshwater transition with comments on the systematics and ecology of the most common diatoms. Diat. Res. 71 : 157-194. 